\title{
Implante de tubo valvulado bioprótese "stentless" em posição aórtica: estudo experimental em ovinos
}

\author{
Paulo César SANTOS*, Luís Roberto GEROLA*, Clodualdo J.N. PESSA*, \\ João Nelson R. BRANCO*, Ivan CASAGRANDE**, Ênio BUFFOLO*
}

RBCCV 44205-576

\begin{abstract}
Santos P C, Gerola L R, Pessa C J N, Branco J N R, Casagrande I, Buffolo E - Implante de tubo valvulado bioprótese "stentless" em posição aórtica: estudo experimental em ovinos. Rev Bras Cir Cardiovasc 2002; 17(1): 90-8.
\end{abstract}

\begin{abstract}
RESUMO: Introdução: O tratamento das lesões valvares representa um grande desafio dentro da cirurgia cardiovascular, pois, até os dias atuais, não temos um substituto ideal para as valvas do coração. As próteses atualmente disponíveis apresentam vantagens e desvantagens; as mecânicas exigem anticoagulação definitiva e as biológicas apresentam baixa durabilidade. Para se chegar a um substituto ideal, são necessárias várias fases, desde a escolha e o preparo do material até o implante em animal de grande porte. Esta última é muito importante, pois a escolha do animal deve ser criteriosa. Ovinos juvenís são animais dóceis de fácil manipulação e suas características anatômicas e biológicas se assemelham muito ao que ocorre com o ser humano e as próteses "stentless" são substitutos mais próximos dos homoenxertos.

Material e Método: Foram operados 30 ovinos juvenís com idade variando de 4,4 a 6,3 meses, peso de 25 a $37 \mathrm{~kg}$, sendo 28 machos. Todos os animais foram submetidos a circulação extracorpórea para implante de bioprótese "stentless" montada em tubo de aorta porcina, com reimplante de artérias coronárias. A operação foi realizada com hipotermia moderada e cardioplegia gelada. O período de seguimento foi de 30 dias e, ao final, realizado ecocardiograma.

Resultados: Houve $8(26,6 \%)$ óbitos no intra-operatório e $6(27,7 \%)$ óbitos no seguimento. O tempo médio de CEC foi de 98 min. Foi realizado ecocardiograma em 16 animais e em nenhum caso foram vistos sinais de endocardite ou outras alterações; a fração média de ejeção foi de $74 \%$.

Conclusão: Este modelo experimental utilizando ovinos para o estudo de biopróteses "stentless" em posição aórtica mostrou-se bem reprodutível e a performance do enxerto bastante factível de ser avaliada, pois ovinos são animais dóceis e de fácil manipulação no seguimento tardio.
\end{abstract}

DESCRITORES: Valva aórtica, cirurgia. Implantle de prótese vascular. Biopróteses.

\section{INTRODUÇÃO}

O tratamento das lesões valvares representa um grande desafio dentro da cirurgia cardiovascular, pois, até os dias atuais, não temos um substituto ideal para as valvas do coração.

Ao contrário das lesões da valva mitral, em que é possível realizar procedimentos conservadores, atuando-se em todas as estruturas anatômicas desde o anel valvar até os músculos papilares; a valva aórtica possui estrutura anatômica mais simples, onde os procedimentos conservadores são menos variados e com resultados pouco satisfatórios quando comparados com as plastias realizadas na valva mitral. Por esta razão, com raras exceções, o tratamento das lesões severas da valva aórtica, quase sempre, representa a troca da mesma.

\footnotetext{
Trabalho realizado na Escola Paulista de Medicina, Universidade Federal de São Paulo. São Paulo, SP, Brasil. Recebido para publicação em setembro de 2001

* Da Disciplina de Cirurgia Cardiovascular da Escola Paulista de Medicina, Universidade de Federal de São Paulo.

** Do Centro de Pesquisa da Labcor. Belo Horizonte, MG.

Endereço para correspondência: Paulo César Santos. Rua Napoleão de Barros, 375, 3ªndar. Disciplina de Cirurgia Cardiovascular. CEP: $04023-062$. Tel: (11) 5576-4055. e-mail: Paulocir@hotmail.com
} 
Santos P C, Gerola L R, Pessa C J N, Branco J N R, Casagrande I, Buffolo E - Implante de tubo valvulado bioprótese "stentless" em posição aórtica: estudo experimental em ovinos. Rev Bras Cir Cardiovasc 2002; 17(1): 90-8.

As próteses valvares atualmente disponíveis têm vantagens e desvantagens; as mecânicas exigem anticoagulação definitiva, com todos os seus inconvenientes, e as biológicas, que não exigem anticoagulação, têm como grande desvantagem a baixa durabilidade.

O estudo de uma prótese biológica inicia-se com a escolha do material, seu preparo e análise experimental; nesta, tentamos reproduzir as condições hemodinâmicas e o stress o mais próximo possível das condições no ser humano. Para chegar a este substituto ideal, são necessárias várias fases de estudo pré-clínico, que incluem in vitro e in vivo.

Os estudos in vitro visam avaliar a performance hemodinâmica e durabilidade das próteses, com testes de fadiga e com duplicadores de pulso que tentam mimetizar aquilo que acontece em território de alta pressão.

Os estudos in vivo devem ser realizados em animais de grande porte, pois somente em animais pode-se observar, com maior fidelidade, aquilo que pode ocorrer com o homem. Nos animais de experimentação, as alterações observadas são aquelas decorrentes da interação dos elementos sangüíneos e imunológicos com o tecido da prótese.

Dentre todos os animais usados para o estudo com próteses valvares cardíacas, citam-se: o implante de tecido valvar em posição subcutânea de ratos. WALTHER et al. (1) implantaram, em cães, próteses em posição mitral; MACKENZIE et al. (2); PAPPAS et al. (3); BIANCO et al. (4) também em posição mitral e, em posição aórtica, LAM et al. (5).

Foram usados também bezerros e suínos para este propósito ${ }^{(6,7)}$ e em ovinos por vários autores ${ }^{(8-}$ 14), mostrando que estes são os animais de grande porte mais usados para estudos experimentais.

Os primeiros estudos experimentais com válvula aórtica foram realizados em aorta descendente de cães ${ }^{(5,16,17)}$.

O primeiro estudo com implante de bioprótese aórtica em posição ortotópica utilizou ovinos juvenís e, para essa finalidade, foi implantada prótese "stentless" em posição subcoronariana (10).

A partir disso, várias técnicas têm-se desenvolvido para elaboração de um modelo experimental ideal para análise desse tipo de prótese cardíaca. A nossa proposição é a criação de um modelo que possa representar de maneira mais próxima do real aquilo que acontece no homem e que seja exeqüível e reprodutível de maneira adequada.

\section{MATERIAL E MÉTODO}

Foram operados 31 ovinos juvenís, com idades variando de 4 a 6,2 meses (média=5.4 meses) e peso médio de $30 \mathrm{~kg}$. Os ovinos eram machos castrados $28(90.6 \%)$ e fêmeas $3(9.4 \%)$ - Tabela 1.
Todos os animais foram avaliados pelo médico-veterinário do centro de pesquisa e, após excluída a presença de anomalias congênitas, problemas respiratórios e infecciosos, o animal permanecia por 15 dias no isolamento. Passado este período de observação e não havendo nenhuma notificação de doença, o animal foi incluído no estudo.

Todos os animais foram vacinados contra tétano (toxóide tetânico 2cc subcutâneo) e anti-parasitários (Invermectin $1 \mathrm{ml} / \mathrm{kg}$ ).

Na véspera da operação, o animal permaneceu em jejum por 12 horas e foi separado dos demais. Foi realizada antibioticoterapia profilática; usamos gentamicina na dosagem de $1 \mathrm{mg} / \mathrm{kg}$ e ampicilina $1 \mathrm{~g}$ intramuscular.

TABELA 1

\begin{tabular}{|c|c|c|c|}
\hline \multicolumn{4}{|c|}{ CARACTERÍSTICAS DOS ANIMAIS } \\
\hline Animal & Sexo & Idade(meses) & Peso( kg) \\
\hline 1 & $\mathrm{~F}$ & 5.53 & 32 \\
\hline 2 & M & 6.03 & 35 \\
\hline 3 & M & 5.85 & 31 \\
\hline 4 & M & 5.83 & 31 \\
\hline 5 & $\mathrm{~F}$ & 5.87 & 32 \\
\hline 6 & M & 5.93 & 35 \\
\hline 7 & M & 5.13 & 31 \\
\hline 8 & M & 5.70 & 31 \\
\hline 9 & M & 4.67 & 29 \\
\hline 10 & M & 4.60 & 28 \\
\hline 11 & M & 4.53 & 28 \\
\hline 12 & M & 5.00 & 28 \\
\hline 13 & M & 4.87 & 30 \\
\hline 14 & M & 5.03 & 29 \\
\hline 15 & M & 4.47 & 30 \\
\hline 16 & M & 5.00 & 27 \\
\hline 17 & M & 5.00 & 31 \\
\hline 18 & M & 4.67 & 28 \\
\hline 19 & M & 5.23 & 30 \\
\hline 20 & M & 4.77 & 27 \\
\hline 21 & $M$ & 5.27 & 30 \\
\hline 22 & M & 5.93 & 31 \\
\hline 23 & $\mathrm{~F}$ & 5.60 & 25 \\
\hline 24 & M & 5.93 & 31 \\
\hline 25 & M & 5.70 & 31 \\
\hline 26 & M & 6.20 & 34 \\
\hline 27 & M & 6.17 & 35 \\
\hline 28 & M & 5.83 & 37 \\
\hline 29 & M & 6.33 & 33 \\
\hline 30 & M & 6.20 & 35 \\
\hline Média & & $5.4 \pm 0.57$ & $30.8 \pm 2.8$ \\
\hline
\end{tabular}


Santos P C, Gerola L R, Pessa C J N, Branco J N R, Casagrande I, Buffolo E - Implante de tubo valvulado bioprótese "stentless" em posição aórtica: estudo experimental em ovinos. Rev Bras Cir Cardiovasc 2002; 17(1): 90-8.

\section{Técnica Operatória}

O animal foi anestesiado com atropina $1 \mathrm{mg}$ e propofol $8 \mathrm{ml}$ endovenoso, além de nova dosagem de antibioticoprofilaxia e metilpredinisolona (250 mg) endovenoso. As drogas anestésicas utilizadas foram o quelicin (100 $\mathrm{mg}$ ) e foi mantida inalação contínua com halotano 1,0 a $1,5 \%$.

Realizou-se toracotomia lateral ao nível do quarto espaço intercostal esquerdo e, uma vez atingindo a cavidade torácica, isolou-se a aorta descendente para a canulação arterial e átrio direito para venosa. Após isto, o animal foi heparinizado com 350 unidades $/ \mathrm{kg}$ e administrado mais $125 \mathrm{mg}$ de metilpredinisolona intravenosa.

A circulação extracorpórea foi iniciada com um fluxo de 50 a $70 \mathrm{ml} / \mathrm{kg} / \mathrm{min}$, o esfriamento é iniciado até a temperatura esofágica atingir 28 graus centígrados.

A partir deste momento, a aorta é pinçada e é feita a cardioplegia anterógrada na raiz da aorta. A dose de solução cardioplégica inicial é de 15 $\mathrm{ml} / \mathrm{kg}$ de solução cristalóide fria a $4^{\circ} \mathrm{C}$, sendo que, a cada 25 minutos, mantemos com de solução cardioplégica sangüínea nos óstios coronarianos $(10 \mathrm{ml} / \mathrm{kg})$.

Após pinçada a aorta, realiza-se uma secção transversal na raiz aórtica, retira-se a cúspides e isolam-se os óstios coronarianos (Figura 1). Após este preparo, iniciamos a sutura do tubo no anel aórtico, a qual é realizada com fio de prolene 5.0 em sutura contínua na parte proximal. Terminada esta fase, iniciamos o reimplante das coronárias, começamos pela coronária esquerda, que é realizada com sutura contínua de prolene 6.0. Para confirmar a perveabilidade, exploramos os óstios com probe de $1.75 \mathrm{~mm}$ e, então, observamos o aspecto final do reimplante dos óstios coronarianos (Figura 2). Por fim, terminamos o procedimento com a anastomose da parte distal do tubo na aorta com sutura contínua de prolene 5.0 (Figura 3).

Terminada a operação, retiramos o ar das cavidades, aberta a pinça da aorta. Após a recuperação dos batimentos cardíacos, iniciamos a ventilação pela máquina e completa-se o aquecimento até $39^{\circ} \mathrm{C}$. Após isto, saímos de circulação extracorpórea e iniciamos o fechamento do toráx por planos.

Cessado o sangramento pelo dreno torácico, este é retirado na própria sala de operação e, com o animal respirando sem ajuda de aparelhos, é extubado e encaminhado para o setor de recuperação pós-operatória.

\section{Pós-Operatório}

\section{Imediato}

O animal permaneceu em regime fechado por 10 a 14 dias, sendo que, neste período, realizam-se antibioticoterapía com $1 \mathrm{~g}$ de ampicilina e $1 \mathrm{mg} / \mathrm{kg}$ de gentamicina IM iniciada, 5 horas após o procedimento operatório e mantido por 36 horas duas vezes ao dia, se o animal apresentar temperatura superior a 40.5 graus centígrados é prolongado por 7 dias.

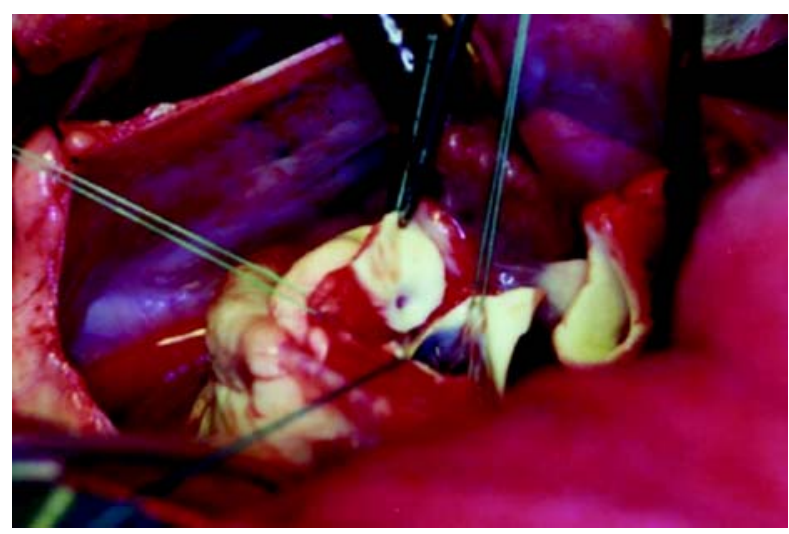

Fig.1 - Individualização dos óstios coronarianos.

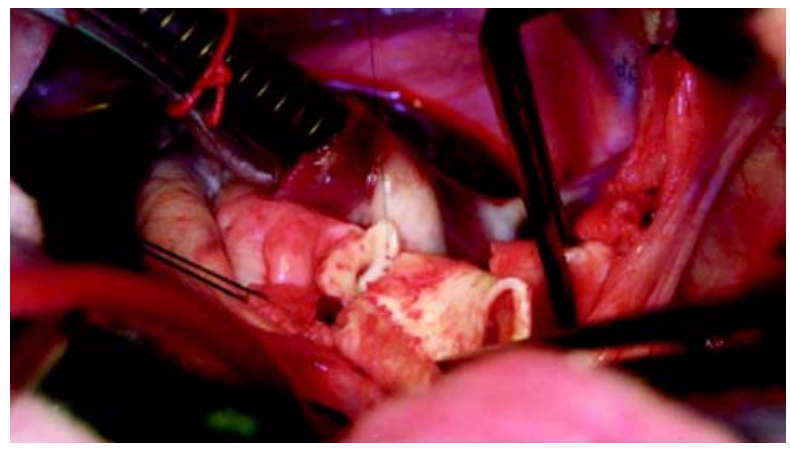

Fig. 2 - Reimplante dos óstios coronarianos.

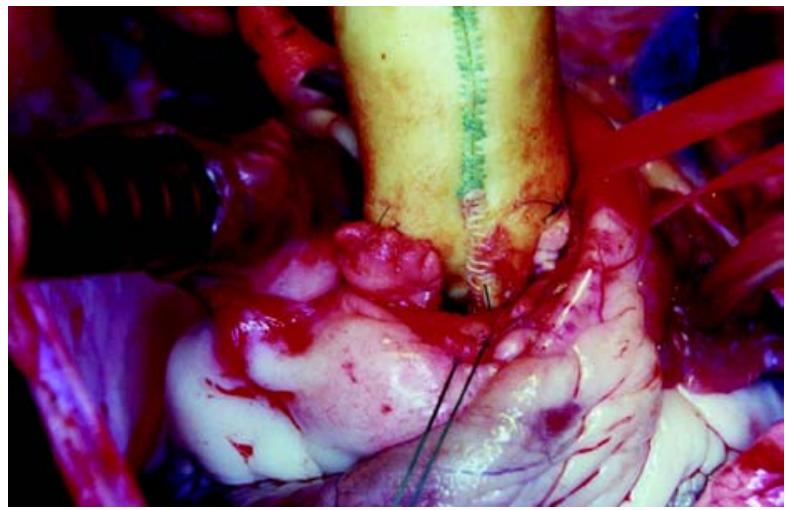

Fig.3 - Aspecto final do implante do tubo stentless. 
Santos P C, Gerola L R, Pessa C J N, Branco J N R, Casagrande I, Buffolo E - Implante de tubo valvulado bioprótese "stentless" em posição aórtica: estudo experimental em ovinos. Rev Bras Cir Cardiovasc 2002; 17(1): 90-8.

Terminado este período de observação, e o animal não apresentar complicações, é transferido para o confinamento localizado no próprio centro de pesquisa, para o seguimento a longo prazo.

\section{Tardio}

Após terminada a fase do pós-operatório imediato, o animal é levado para o setor de confinamento. Este local consiste de um ambiente que possua alimentação adequada e balanceada para a idade do ovino, e também é coletado o sangue para controle da função renal, hematológica e bioquímica, na primeira semana e no primeiro mês.

Ao animais são soltos no pasto para desenvolverem atividade física sem restrição.

\section{Ecocardiograma}

Foram feitos, em todos os animais que sobreviveram ao primeiro mês, avaliação do funcionamento da válvula, da fração de ejeção e do gradiente transvalvar. O aparelho usado foi um Ultramark-6, com um transdutor de 3.5 e $2.5 \mathrm{mhz}$.

\section{Biopróteses "Stentless"}

São próteses biológicas feitas sem suporte em um tubo de aorta heteróloga. Estas próteses chegam até nós montadas. São coletadas a fresco de aorta de porco. A partir daí, são transportadas em solução gelada de 4 a $8^{\circ} \mathrm{C}$ em solução salina tamponada, com $\mathrm{pH}$ de 7.4, o tampão usado é o fosfato. Posteriormente, estas próteses são tratadas com solução de glutaraldeído a $0,4 \%$ por um período de 8 semanas. Após este período, são esterilizadas com fosfato, glutaraldeído a 0,6\% e etanol a $20 \%$ e, por fim, embaladas em solução de glutaraldeído a $0,2 \%$. Todo este processo é realizado na fábrica da Labcor, em Belo Horizonte.

\section{RESULTADOS}

Foram realizadas 31 operações de implante de tubo valvulado "stentless", em posição subcoronária, com reimplante das coronárias em carneiros juvenís com idade média de 5.2 meses, peso médio no momento da operação era de $25 \mathrm{~kg}$.

Houve 8 mortes intra-operatórias com, taxa de mortalidade de $25 \%$. Três desses casos foram por sangramento incontrolável na linha de sutura distal, 2 outros não recobraram nível de consciência após o término da operação e tiveram que ser sacrificados, 1 apresentou infarto agudo do miocárdio e não manteve condições hemodinâmicas estáveis fora de extracorpórea e os últimos 2 morreram por hipóxia refratária, ao término da operação.
Entre os animais restantes, 23 foram acompanhados clinicamente por um período de 30 dias. Neste período, houve 3 mortes, um animal teve que ser sacrificado por paralisia irreversível dos membros posteriores, o outro teve endocardite da prótese e foi encontrado morto e um terceiro apresentou morte súbita.

Ao final, foram acompanhados, por um período de 30 dias, 20 carneiros e com uma taxa de mortalidade global de $37.8 \%$, sendo que a taxa de mortalidade, no seguimento, foi de $14 \%$.

As próteses usadas foram de tamanhos $19 \mathrm{em}$ 16 casos, 21 em 9 casos e 23 no restante.

Todos os animais apresentaram, ao final de 1 mês, ganho de peso adequado para a espécie.

Os valores normais de literatura para alguns elementos da bioquímica e hematológicos são:

- Creatinina-0.6 a $1.5 \mathrm{mg} / \mathrm{dl}$.

- Potássio- 4.1 a $5.8 \mathrm{mmol} / \mathrm{l}$

- Sódio- 141 a $151 \mathrm{mmol} / \mathrm{l}$

- DHL- 504- $1049 \mathrm{U} / \mathrm{l}$

- TGP- 5 a $17 \mathrm{U} / \mathrm{l}$

- TGO- 40 a $96 \mathrm{U} / \mathrm{l}$

- CPK- 8 a $100 \mathrm{U} / \mathrm{I}$

Os valores de elementos da coagulação são semelhantes àqueles encontrados no homem.

Os níveis TGO e TGP se mantiveram elevados acima do normal em 11 animais e retornando ao normal em 7 deles, um mês após a operação, o valor médio de TGO foi de $126.6 \pm 59.5$ caindo para $118.9 \pm 67.7$ ao final de 30 dias, e de TGP $12.6 \pm 6.5$ com discreto aumento para $14.1 \pm 5.8$ nesse mesmo período.

Não houve alterações significativas nos valores de uréia e creatinina; o valor encontrado para uréia foi de $59.0 \pm 14.6$ e com 30 dias $54.7 \pm 14.0$, e valor para creatinina $0.6 \pm 0.12$ e ao final queda para $0.5 \pm 0.08$.

Para avaliar o grau de isquemia do coração, analisamos os resultados de creatininofosfoquinase e desidrogenase láctica. Apenas 2 animais apresentaram níveis elevados de CPK e DHL no pós-operatório, com uma semana, os níveis de CPK média de $94.9 \pm 62.3$ e reduzindo para $56.7 \pm 28.0$, os valores médios iniciais de DHL foi de $450 \pm 155$ reduzindo para $448 \pm 113$.

No que se refere aos elementos hematológicos, 15 animais mantiveram $\mathrm{Ht}$ abaixo de $30 \%$, sendo que todos recuperaram em um mês, 
Santos P C, Gerola L R, Pessa C J N, Branco J N R, Casagrande I, Buffolo E - Implante de tubo valvulado bioprótese "stentless" em posição aórtica: estudo experimental em ovinos. Rev Bras Cir Cardiovasc 2002; 17(1): 90-8.

sendo o valor médio de $26.1 \pm 5.6$ e ao final aumentou para $33.8 \pm 4.0 .0$ valor médio de hemoglobina inicial foi de $8.5 \pm 1.6$ e aumentou para $11.0 \pm 1.3$, como mostrado nas Tabelas 7 e 8. Treze animais apresentaram leucocitose, sendo 2 deles importante com número de leucócitos acima de 20.000 e estes se mantiveram mesmo após 1 mês de observação, porém os valores médios iniciais foram de $13.000 \pm 4500$ em $\mathrm{cm}^{3}$ e final de $13.300 \pm 6.800$.

No que diz respeito à coagulação (Tabelas 10 e 11), o número médio de plaquetas foi de $297.7 \pm$ 62.2 e elevou-se para $410.0 \pm 67.2$ ao fim de 30 dias e nem alteração de tempo de protrombina, cujos valores iniciais médios foram de $35.1 \pm 6.4$, e 35.7 \pm 6.2 ao final de 30 dias.

Os valores ecocardiográficos encontrados nos mostraram bom desempenho hemodinâmico das válvulas e do músculo cardíaco, não foram encontradas áreas acinéticas ou hipocinéticas, a fração de ejeção média foi de $74 \% \pm 5.7$ e o gradiente médio de $3.9 \pm 2.2$, diâmetro diastólico de $4.1 \pm 1.0$. Apenas duas válvulas apresentaram grau de insuficiência discreto, e um gradiente médio de $4 \mathrm{mmHg}$, no total das válvulas. Nenhuma das válvulas apresentava sinais de endocardite ou perfuração dos folhetos (Tabela 2).

O tempo médio de circulação extracorpórea foi de $97.1 \pm 24.3$ minutos e o tempo de pinçamento aórtico de 71.8 minutos, o total de mortes no intra-operatório e no seguimento mostrado no Gráfico 1.
GRÁFICO 1

Gráfico da mortalidade em relação ao ano de carneiro operados

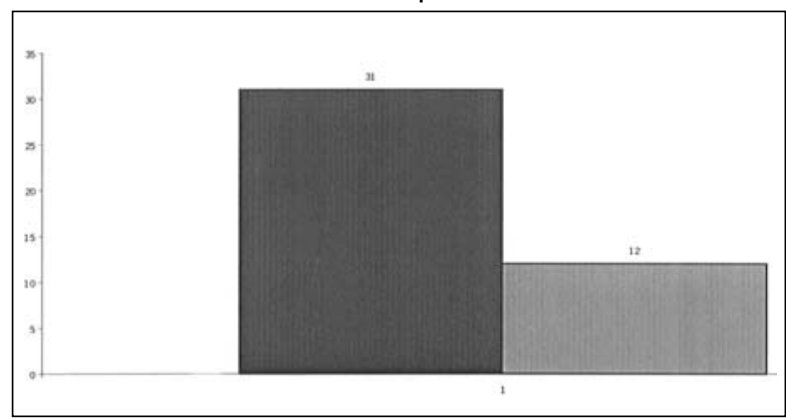

\section{COMENTÁRIOS}

A mais importante fase de estudo pré-clínico, em testes de próteses cardíacas, envolve o implante dessas válvulas em posição ortotópica em animais de grande porte. Durante esta fase do estudo, características da performance da válvula, bem como a sua segurança, são avaliadas e também as configurações da prótese, da técnica de implante e o estudo hemodinâmico in vivo. Além disso, a durabilidade global terá que ser bem analisada e suas complicações, o tromboembolismo, calcificação, hemólise, trombos e doenças cardíacas e sistêmicas.

Com isso, torna-se imperativo o uso de um modelo experimental adequado e que melhor possa pressupor aquilo que acontece em humano.

TABELA 2

VALORES ECOCARDIOGRÁFICOS

\begin{tabular}{cccccc}
\hline Ident. & F.E (\%) & Gradiente médio & DD(cm) & Espessura do septo & Espessura da pp \\
\hline 1 & 67 & 4.2 & 4.6 & 1.0 & 1.0 \\
2 & 65 & 1.6 & 4.7 & 0.7 & 0.7 \\
3 & 75 & 5.3 & 3.6 & 0.8 & 0.8 \\
4 & 75 & 8.2 & 4.4 & 0.6 & 0.6 \\
5 & 79 & 3.0 & 5.3 & 0.7 & 0.7 \\
6 & 73 & 9.6 & 4.4 & 0.7 & 0.7 \\
7 & 72 & 4.1 & 3.7 & 0.7 & 0.7 \\
8 & 77 & 1.8 & 6.3 & 0.8 & 0.7 \\
9 & 84 & 1.7 & 3.3 & 0.6 & 0.6 \\
10 & 72 & 1.4 & 4.6 & 0.7 & 0.8 \\
11 & 68 & 3.0 & 2.5 & 0.6 & 0.6 \\
12 & 68 & 1.5 & 3.3 & 0.8 & 0.6 \\
13 & 78 & 4.8 & 4.3 & 0.9 & 0.7 \\
14 & 82 & 3.4 & 3.7 & 0.7 & 0.9 \\
15 & 80 & 4.5 & 4.3 & 0.8 & 0.7 \\
16 & 67 & 4.8 & 2.3 & 0.6 & 0.7 \\
17 & 77 & 3.6 & 5.3 & 0.7 & 0.6 \\
\hline Dédia & 74.0 & 3.9 & 4.1 & 0.1 & 0.7 \\
\hline
\end{tabular}


Santos P C, Gerola L R, Pessa C J N, Branco J N R, Casagrande I, Buffolo E - Implante de tubo valvulado bioprótese "stentless" em posição aórtica: estudo experimental em ovinos. Rev Bras Cir Cardiovasc 2002; 17(1): 90-8.

Essas próteses são testadas em duplicadores de pulso e em testes de estresse que tentam simular aquilo que ocorre no homem ${ }^{(18)}$.

Uma outra forma de avaliar estas próteses é seu implante em aorta descendente de cães, que apesar de ser um procedimento relativamente mais simplificado, é um modelo sujeito a críticas principalmente pelo fato de manter a válvula nativa, o que produziria resultados não muito confiáveis além de estar numa posição anatômica desfavorável, alguns autores iniciaram os primeiros procedimentos com este modelo experimental (19-21).

A outra forma de avaliar este tipo de próteses era o implante em outros locais, com tecido subcutâneo de ratos, em posição mitral ou tricuspídea em cães e carneiros. Contudo, estas próteses também eram avaliadas em posição mitral, o que não mimetiza aquilo de acontece em território aórtico, daí não se pode concluir diversos aspectos biomecânicos destas próteses.

Têm-se usado várias espécies de animais com esse propósito; alguns autores ${ }^{(8,4,10,13,17,22)}$ citam experimentos realizados com cavalos, ratos, cabras, porcos e cães.

O uso de cabras tem sido relatado com sucesso, porém, em várias áreas, a utilização deste animal é limitada, o que torna seu uso pouco difundido(22). Os cães, são usados mais amplamente, devido à facilidade de obtenção; porém a incidência de endocardite e de outras doenças sistêmicas é maior.

Suínos apresentam várias dificuldades, entre elas a presença de variações anatômicas ${ }^{(25)} \mathrm{com}$ plicações pós circulação extracorpórea, dificuldades para acesso venoso periférico e o manuseio e cuidados com a saúde bastante difíceis ${ }^{(26)}$.

A necessidade de cuidados especializados e 0 risco de transmissão de doenças para o pessoal de laboratório é grande, principalmente hepatite e micobactérias; daí o uso bastante limitado de primatas como macaco e babuínos(27).

Vários autores têm usado ovinos como modelo experimental, para o implante de próteses cardíacas e concluíram que carneiros jovens são modelos ideais para este tipo de procedimento, pois, a calcificação nas próteses pode ser avaliada com mais acurácia durante o seu desenvolvimento(12)

Analisando todos estes animais, percebemos que o melhor modelo para avaliação de próteses cardíacas, tanto a longo quanto a curto prazo, são carneiros jovens, visto que são animais, fáceis de serem cuidados no seguimento a longo prazo, características de desenvolvimento pôndero-estatural adequado e em curto prazo de tempo.
Nosso experimento partiu do princípio de que o melhor local para se avaliar uma prótese seria o seu local originalmente anatômico. Para isso, a prótese deveria ser implantada em posição ortotópica.

Os ovinos em comparação com outros animais, como por exemplo, porcos, suportam melhor as alterações produzidas pela circulação extracorpórea ${ }^{(7)}$. O início da circulação extracorpórea e a manutenção foi de maneira convencional para o peso do animal, com fluxo arterial adequado para manter bom débito cardíaco e boa oxigenação, sendo que a administração das demais drogas usadas durante o procedimento foi feita de maneira já consagrada e usada por outros autores ${ }^{(10-13)}$.

A solução cardioplégica inicialmente usada era cristalóide fria na raiz da aorta e a manutenção era feita coma a mesma. A partir da quarta operação, passamos a fazer a dose de manutenção com solução cardioplégica sangüínea nos óstios coronarianos, e observamos uma melhor recuperação dos batimentos cardíacos após o despinçamento da aorta. Isto contribuiu favoravelmente para a diminuição do tempo de CEC, pois, com esta recuperação mais rápida da função cardíaca, foi mais fácil sair de perfusão.

De maneira geral, a técnica de implante do tubo valvulado "stentless" em posição subcoronariana, a não ser certas peculiaridades, como o coto proximal de aorta deixado no anel para o reforço da sutura, o demais passos não diferem muito da literatura convencional deste tipo de operação em humanos.

Esta técnica de implante de prótese aórtica "stentless" em posição ortotópica, com reimplante das coronárias, nos mostrou um grande leque de variedades no tamanho das próteses usadas, sendo que usamos de 21 a 25. Desta maneira, o anel aórtico é bem exposto e proporciona este fato. $O$ anel aórtico do carneiro é o que, proporcionalmente, mais se aproxima do anel aórtico do homem, em tamanho ${ }^{(28)}$. Por isso, a importância na diversidade do tamanho das próteses utilizadas. DAVID et al. (10) utilizaram próteses em posição aórtica todas de tamanho 21 , sem reimplante de coronárias, OUYANG et al. (12) descreveram o implante de próteses "stentless" em posição ortotópica aórtica em carneiros juvenís, e utilizaram próteses de tamanho $19 \mathrm{em}$ todos os casos.

Ocorreram 8 óbitos no intra-operatório, uma mortalidade de $25,8 \%$. Em 4 destes casos ocorreu na primeira semana do estudo e, nos primeiros casos, em 2 por sangramento incontrolável na linha de sutura proximal da aorta, a qual foi feita com pontos separados e, mesmo depois da protaminização, não houve formação suficiente de coágulo para cessar a hemorragia; em outro, o tempo de CEC foi muito prolongado e o coração não recuperou os batimentos e, no último caso, por infarto trans-operatório traduzido por supradesnível do segmento ST ao monitor cardíaco, além de enrijecimento da parede ventricular esquerda, provavelmente por torção na coronária esquerda. 
Santos P C, Gerola L R, Pessa C J N, Branco J N R, Casagrande I, Buffolo E - Implante de tubo valvulado bioprótese "stentless" em posição aórtica: estudo experimental em ovinos. Rev Bras Cir Cardiovasc 2002; 17(1): 90-8.

Os outros 4 casos ocorreram durante a segunda semana do estudo, 2 animais apresentaram, após sair de CEC, hipóxia grave e refratária, com saída de grande secreção amarelada pela cânula traqueal, a presença de secreção amarelada sugere algum processo infeccioso recente, que, apesar de todo o cuidado pré-operatório, este fato não foi identificado; entre outras causas para esta intercorrência, cita-se a insuficiência da prótese levando a edema pulmonar agudo, acúmulo de secreções por desenvolvimento de atelectasias, provocada pela circulação extracorpórea. Os outros 2 animais não apresentaram qualquer intercorrência durante a operação, porém, terminado procedimento, não recobraram o nível de consciência, mesmo após um período de 6 horas após o término da operação, em ventilação mecânica. As possíveis causas para esta intercorrência são, principalmente, embolia área maciça, fluxo cranial insuficiente e redirecionamento da cânula arterial para caudal. Portanto, estes 2 animais tiveram que ser sacrificados. As pupilas encontravam-se paralíticas e midriáticas, além de ausência total de reflexos motores e respiratórios.

Durante o seguimento, houve 3 mortes, daqueles 21 restantes, perfazendo uma mortalidade de $14,7 \%$ em 30 dias. Um animal apresentou paralisia completa dos membros posteriores e teve que ser sacrificado no 6을 $\mathrm{PO}$; este animal apresentou um tempo de CEC extremamente elevado com duração de 151 minutos, pois apresentou uma grave intercorrência, que foi uma lesão na coronária direita e a correção foi muito difícil, com um pinçamento aórtico de 98 minutos. Isto, deve ter sido o fato que levou um baixo fluxo por um tempo grande para as extremidades distais. O outro animal foi encontrado morto no 27으 PO e, ao exame macroscópico da prótese, apresentava-se com aspecto de endocardite, com destruição do tecido protético, e o outro animal

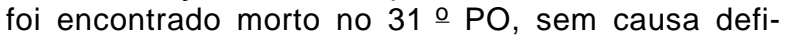
nida, pois a prótese apresentava-se normal e não havia áreas de infarto, porém o coração estava bastante aumentado.

A baixa mortalidade durante o seguimento pósoperatório e a pequena ocorrência de processos infecciosos nos mostram a viabilidade de cuidar destes animais na área de confinamento. $\mathrm{E}$, além disso, a técnica de reimplante dos óstios coronarianos não foi causa imediata de óbito no seguimento. Em outros modelos, a taxa de infecção e septicemia são maiores, chegando a $50 \%(21)$; isto se deve a freqüentes bacteremias que ocorrem em cães, inerentes ao hábito de vida destes animais.

De maneira global, a mortalidade foi de 11 carneiros em 31 operados, com uma mortalidade geral de 35,4\%, com curva atuarial de 30 dias de 64.6\%. ALEXI-MESKISHVILLI et al. (28) descreveram o trabalho de Nikolai Terebinski, que realizou 250 operações valvares em cães, com uma taxa de mortalidade de $34 \%$, quando na valva tricúspide e
$52 \%$ na valva mitral. ARBUSTIN et al. (8) obtiveram uma taxa de mortalidade de $16,6 \%$, realizando operações sobre a valva tricúspide de carneiros; BARNHART et al. (29), com mesmo modelo experimental, obtiveram uma taxa de mortalidade de $20 \%$. BIANCO et al. (4) testaram o cão como modelo experimental, para a análise a longo prazo de próteses cardíacas; obtiveram uma taxa de mortalidade de $18 \%$; BJÖRK et al. (22), operando cabras, obtiveram uma taxa de mortalidade de $29 \%$, em posição mitral.

Os estudos que utilizaram cães, realizando as operações sobre a valva aórtica, apresentaram uma taxa de mortalidade maior. LOWER et al. (21) obtiveram uma taxa de mortalidade de $53,3 \%$, com implante de prótese aórtica na aorta descendente de cães. BROWN et al. (30) estudaram 13 carneiros, nos quais implantaram as próteses em posição supra coronária, obtiveram uma taxa de mortalidade de $69 \%$. DAVID et al. (10) foram os primeiros a implantar próteses "stentless", em posição aórtica em carneiros velhos, obtiveram uma taxa de mortalidade operatória de $25 \%$ e global de $50 \%$. SALERNO et al. (13) operaram 3 carneiros adultos com implante de prótese aórtica metálica, com $0 \%$ de mortalidade. OUYANG et al. (12) desenvolveram um modelo experimental para o implante de prótese aórtica "stentless", em posição ortotópica para troca de valva aórtica, para isso, operaram 27 carneiros juvenís com uma taxa de mortalidade de $48 \%$. A operação foi feita de maneira convencional, sem reimplante dos óstios coronarianos.

Este último estudo é o que mais se aproxima da técnica por nós utilizada, porém, como já mostrado, usamos próteses de diferentes tamanhos e a mortalidade global, em nosso estudo, foi menor. Os outros estudos com operações sobre valvas tricúspide e mitral, as taxas de mortalidade são menores, porém para a avaliação de próteses em posição aórtica deve ser feita na sua posição ortotópica, levando, com isso, maior confiabilidade dos resultados obtidos para que possamos inferir aquilo de poderá ocorrer no homem.

O controle laboratorial foi analisado na primeira semana do pós-operatório e no trigésimo pós-operatório. Não foram realizadas as dosagens no pré-operatório, por questões logísticas. Cuntudo, os valores obtidos, nestas duas seqüências citadas, nos permita avaliar as principais alterações que poderiam ocorrer.

Foram estatisticamente significativos os valores da redução da creatinina e uréia, o que pode ser explicado pelas possíveis alterações induzidas pela CEC. Estes valores, porém, se mantiveram no limite da normalidade, o que nos leva a analisar que a perfusão renal foi adequada, bem como a não ocorrência de baixo débito cardíaco. Em outros estudos, não houve alterações nestes valores $(4,12,13)$.

Para obtermos uma análise funcional da prótese, bem como da função cardíaca, conseguimos realizar ecocardiograma em 17 animais. O valor médio da fração de ejeção obtido, em torno de $74 \%$, nos 
Santos P C, Gerola L R, Pessa C J N, Branco J N R, Casagrande I, Buffolo E - Implante de tubo valvulado bioprótese "stentless" em posição aórtica: estudo experimental em ovinos. Rev Bras Cir Cardiovasc 2002; 17(1): 90-8.

mostra a boa função cardíaca e um bom desempenho da prótese, e um gradiente médio da via de saída do ventrículo esquerdo de $3,9 \mathrm{mmHg}$ e um diâmetro diastólico final de $44,9 \mathrm{~mm}$. Procuramos comparar estes dados com valores normais de ecocardiograma para carneiros juvenís sem nenhuma doença pregressa. Porém não encontramos dados na literatura a este respeito. Por isso, na tentativa de acharmos parâmetros para a normalidade, realizamos 3 exames em carneiros sadios imediatamente antes da operação e os valores não diferiram daqueles encontrados nos animais do experimento. A fração de ejeção ficou em torno de $75 \%$, o gradiente médio em torno de $3 \mathrm{mmHg}$ e o diâmetro diastólico final em $40 \mathrm{~mm}$.

Os dados do ecocardiograma nos permite analisar a prótese e seu funcionamento e, indiretamente, o grau de isquemia desse coração, porém, para obtermos dados extremamente confiáveis em relação ao reimplante dos óstios coronarianos, é necessária a realização de estudo coronariográfico.

Sendo de grande importância, o estudo préclínico, em animais de grande porte, é imperativo que se faça um modelo experimental adequado.

Ovinos juvenís, com idade entre 4 e 6 meses, demonstram serem modelos excelentes em relação a facilidade e a reprodutibilidade da técnica empregada. E mais importante ainda é o acompanhamento a longo prazo destes animais; como são carneiros juvenís, seu rápido desenvolvimento nos permite, a curto prazo, analisar as alterações que possam ocorrer neste tipo de prótese. Além disso, há facilidade na observação clínica e na coleta de exames, o que não provoca estresse nestes animais.

\section{CONCLUSÕES}

- O ovino juvenil, como modelo experimental para análise de prótese cardíaca, mostrou-se bastante eficaz, para a conclusão de um estudo, para uma posterior aplicabilidade clínica para este tipo de prótese, e além disso os ovinos são animais dóceis e fáceis de manipular no pósoperatório imediato e tardio em relação à alimentação e aos cuidados no confinamento e controle clínico.

- Biopróteses aórticas “stentless", quando implantadas em posição ortotópica, têm maior confiabilidade para avaliação hemodinâmica e estrutural.

- Um estudo a longo prazo permitirá avaliar a anatomia das coronárias após o implante destas.

RBCCV 44205-576

Santos P C, Gerola L R, Pessa C J N, Branco J N R, Casagrande I, Buffolo E - A juvenile sheep model for the stentless bioprostheses implanted as aortic root replacements. Rev Bras Cir Cardiovasc 2002; 17(1): 90-8.

ABSTRACT: Introduction: Often, studies on aortic prosthetic valves analyze their performance not at the primitive position, but using tricuspid and mitral ones or inserting it in the descending aortic area. Taking that into account, it would be relevant to observe results in those studies in which the conclusions are based on their original implantation.

Material and Methods: Thirty young sheep, 28 males and 2 females, were operated on. Ages ranged 4.4 to 6.3 months and weights 25 to $37 \mathrm{Kg}$. Extra corporeal circulation (ECC) was applied to all the animals, always guided by the conventional criteria, that is to say, the arterial cannula was inserted in the thoracic aorta just past the arterious ducts and the venous cannulae, a single one, was placed in the right atrium. The operation was performed according to the principle of moderately low body temperature$29^{\circ} \mathrm{C}$ - and cold cristalloid cardioplegia, in the aortic root, to induce; but otherwise was utilized blood to the maintenance. Following with the technique proposed, the aortic valve of the animal heart was completely excised and finally implanted the valved tube stentless, not forgetting the refixation of the coronary main. The sheep were kept in clinical and laboratorial observation during thirty days in what was included a echocardiogram in the end.

Results: The results demonstrated 8 intraoperatory deaths (26.6\%), and 6 during the following (27.7\%). The mean time of ECC was 98 minutes. The dimension of the majority prothetics valves were 21 and 23. Only two valves evoluted with mild insufficiency, none denoted evidences of the endocarditis neither of leaflets perfuration, the mean fraction of ejection was $74 \%$, the left ventricle had its wall preserved in all cases.

Conclusion: This experimental model can certainly reproduce nearly the reality, confirming good parameters of prothetics stentless evaluation in the aortic position. And the sheep declared itself to be pleasant, which helps us its control during the whole study.

DESCRIPTORS: Aortic valve surgery. Blood vessel prosthesis, implantation. Bioprosthesis. 
Santos P C, Gerola L R, Pessa C J N, Branco J N R, Casagrande I, Buffolo E - Implante de tubo valvulado bioprótese "stentless" em posição aórtica: estudo experimental em ovinos. Rev Bras Cir Cardiovasc 2002; 17(1): 90-8.

\section{REFERÊNCIAS BIBLIOGRÁFICAS}

1 Walther T, Falk A, Diegeler A et al. - Effectiveness of different anticalcification treatments for stentless aortic bioprostheses. Thorac Cardiovasc Surg 1999; 47: 23-5.

2 Mackenzie M B, Titus J L, Ellis F H, Pappas G - Experimental semilunar valve homografts: morphologic features. J Thorac Cardiovasc Surg 1965; 50: 410-20.

3 Pappas G, Titus J L, Berghuis J, Mckenzie M B, Ellis $\mathrm{F} \mathrm{H} \mathrm{Jr.} \mathrm{-} \mathrm{Dog} \mathrm{mitral} \mathrm{valve} \mathrm{homografts} \mathrm{and} \mathrm{heterografts.}$ Surg Forum 1966; 17: 175-6.

4 Bianco R W, St Cyr J A, Schneider J R et al. - Canine model for long-term evaluation of prosthetic mitral valves. J Surg Res 1986; 41: 134-40.

5 Lam C R, Aram H H, Munnel E R - An experimental study of aortic valve homografts. Surg Gynecol Obst 1952; 94: 129-35.

6 Yarbrough J W, Roberts W C, Reis R L - Structural alterations in tissue cardiac valves implanted in patients and in calves. J Thorac Cardiovasc Surg 1973; 65: 364-75.

7 Hazekamp M G, Goffin Y A, Huysmans H Á - The value of the stentless biovalve prosthesis: an experimental study. Eur J Cardiothorac Surg 1993; 7: 514-9.

8 Arbustin E, Jones M, Ferrans V J - Formation of cartilage in bioprosthetic cardiac valves implanted in sheep: a morphologic study. Am J Cardiol 1983; 52: 632-6.

9 Thiene G, Laborde F, Valente $\mathrm{M}$ et al. - Experimental evaluation of porcine-valved conduits processed with a calcium-retarding agent (T6). J Thorac Cardiovasc Surg 1986; 91: 215-24.

10 David T E, Ropchan G C, Butany J W - Aortic valve replacement with stentless porcine bioprostheses. $J$ Card Surg 1988; 3: 501-5.

11 David T E, Bos J, Ropchan G C, Pollick C. A stentless porcine aortic bioprosthesis for aortic valve replacement. Surg Heart Valve Dis 1990; 18: 828-35.

Ouyang D W, Salerno C T, Pederson T S, Bolman R M 3rd, Bianco R W - Long-term evaluation of orthotopically implanted stentless bioprosthetic aortic valves in juvenile sheep. J Invest Surg 1998; 11: 175-83.

13 Salerno C T, Pederson T S, Ouyang D W, Bolman R M 3rd, Bianco R W - Chronic evaluation of orthotopically implanted bileaflet mechanical aortic valves in adult domestic sheep. J Invest Surg 1998; 11: 341-7.

14 Flameng W J, Ozaki S, Yperman J et al. - Calcification characteristics of porcine stented valves in a juvenile sheep model. Ann Thorac Surg 2001; 71: S401-5.

15 Trantina-yates A, Weissenstein C, Human P, Zilla P Stentless bioprosthetic heart valve research: sheep versus primate model. Ann Thorac Surg 2001; 71 (5 suppl): S422-7.
16 Hufnagel C A - Aortic plastic valvular prosthesis. Bull Georgtown Univ Med Center 1952; 4: 128.

17 Murray G - Homologous aortic valve segment transplant as surgical treatment for aortic and mitral insufficiency. J Thoracic Surg 1955; 30: 633-7.

18 Nagy Z L, Fisher J, Walker P G, Watterson K G - The effect of sizing on the in vitro hydrodynamic characteristics and leaflet motion of the Toronto SPV stentless valve. J Thorac Cardiovasc Surg 1999; 117: 92-8.

19 Pritchard G R, Wright M B, Johnston J B - Transplantation of heart valve cusps: an experimental comparison of homografts and heterografts. J Thorac Cardiovasc Surg 1967; 53: 322-6.

20 Mohri H, Reichenbach D D, Barnes R W, Nelson R J, Merendino K A - Studies of antigenicity of the homologous aortic valve. J Thorac Cardiovasc Surg 1967; 54: 564-72.

21 Lower R R, Stoffer R C, Shumway N E - Autotransplantation of the pulmonic valve into the aorta. $J$ Thorac Cardiovasc Surg 1960; 39: 680-7.

22 Björk V O \& Sternlieb J - Artificial heart valve testing in goats. Scand J Thorac Cardiovasc Surg 1986; 20: 97-102.

23 Monro J L, Gavin J B, Barrat-boyes B G - A comparison of antibiotic-sterilized, stent-mounted pulmonary and aortic valve allografts in the mitral region of dogs. Thorax 1974; 29: 323-8.

24 Tector A J, Boyd W C, Korns M E - Aortic valve allograft rejection. J Thorac Cardiovasc Surg 1971; 62: 592601.

25 Swan H \& Meagher D M - Total body bypass in miniature pigs: postperfusion pulmonary hypertension. J Thorac Cardiovasc Surg 1971; 61: 956-67.

26 Swan H \& Piermattei D L - Technical aspects of cardiac transplantation in the pig. Ann Thorac Surg 1971; 61: 710-23.

27 Sands M P, Rittenhouse E A, Mohri H, Merendino K A An anatomical comparison of human, pig, calf and sheep aortic valves. Ann Thorac Surg 1969; 8: 401-14.

28 Alexi-Meskishvilli V V, Potapov V E, Beyer E A, Hetzer $\mathrm{R}$ - Nikolai Terebinski: a pioneer of the open valve operation. Ann Thorac Surg 1998; 66:1440-3.

29 Barnhart G R, Jones M, Ishihara T, Chavez A M, Rose $D M$, Ferrans $\vee \mathrm{J}$ - Failure of porcine aortic and bovine pericardial prosthetic valves: An experimental investigationin young sheep. Circulation 1982; 66; (2 Pt 2) 150-3.

30 Brown W M, Jay J L, Gott J P et al. Placement of aortic valve bioprostheses in sheep via a left thoracotomy. Implantation of stentless porcine heterografts. Trans Am Soc Artif Intern Organs 1991; 37: M445-6. 